\title{
Cancer du sein : évolution des dogmes
}

\section{Breast Cancer: Evolving Concepts}

\section{A. Lesur - F. Guillemin}

(C) Lavoisier SAS 2019

Quoi que l'on fasse, le cancer du sein reste à travers le temps le premier cancer en incidence chez la femme, lentement mais inexorablement toujours en augmentation (chiffres INCa : 58968 nouveaux cas de cancer du sein en France métropolitaine en 2017, 11883 décès estimés en 2017).

Hormis le pourcentage élevé de population que cela touche, cette maladie pose sans cesse de nouvelles questions, non pas tant en organisation de traitements (cette localisation est le meilleur exemple d'illustration de la notion de parcours mais surtout en choix thérapeutiques).

Si bien qu'en ce début de 2019, nous avons choisi de façon éclectique quelques thèmes actuels qui, à la fois, questionnent et préoccupent.

Il est fini et bien fini le temps d'avant les Plans cancers où une boule dans le sein se voyait opérée pour débarrasser la patiente - qui n'attendait que cela, croyant résoudre le problème - et faire un diagnostic souvent déconcertant, imposant des lendemains complexes car non anticipés. Plus personne n'imaginerait traiter une patiente d'un probable cancer du sein sans connaître ses caractéristiques anatomopathologiques et immunohistochimiques. Cela conditionne tout le temps diagnostique parfois trop long pour la patiente, mais qui optimise la prise en charge ultérieure en pertinence.

À travers les décennies, la désescalade dans la morbidité des traitements n'a cessé d'être une règle d'or. Ainsi, les chirurgiens n'ont pas attendu E. Winer à San Antonio en décembre dernier : « the challenge of doing less » pour appliquer la devise du Bachaus less is more passés du Halstedt au Patey pour arriver à la mastectomie partielle associée à la radiothérapie mammaire, les chirurgiens ont peu à peu, avec prudence néanmoins, laissé au ganglion sentinelle (GS) une

\footnotetext{
A. Lesur $(\bowtie) \cdot$ F. Guillemin

Service parcours sein, institut de cancérologie de Lorraine, 6, avenue de Bourgogne, CS 30519,

F-54519 Vandœuvre-lès-Nancy cedex, France

e-mail : a.lesur@nancy.unicancer.fr

F. Guillemin

Institut de cancérologie Jean-Godinot,

1, rue du Général-Koenig-CS,

F-51726 Reims cedex, France
}

place grandissante, réservant les curages axillaires aux situations incontournables (Il n'y a pas si longtemps, un numéro entier de cette revue était consacré au sujet (Le creux axillaire - Oncologie (2013) Volume 15 (6) : 275-339).

Ces notions posent la question du bilan initial qui bien qu'indispensable est souvent mal vécu, car retardant le début du traitement tant attendu une fois le diagnostic de malignité établi.

Faut-il privilégier un bilan iconographique locorégional pointu dans les mains de radiologues experts pour se donner toutes les chances d'opérer au bon endroit et de la meilleure façon ? Tant que tout GS atteint (micro- ou macrométastase) faisait l'objet d'une reprise chirurgicale par curage, il n'y avait aucun doute pour considérer que cela représentait un avantage certain (la pratique de la technique OSNA nous en avait persuadés). Mais voici que se posent deux questions incontournables :

- la fiabilité de l'exploration mammaire et axillaire estelle telle qu'il faille privilégier relecture, confrontation radiochirurgicale, nouveaux prélèvements et potentiel retard apporté au geste curatif ? Si tel est le cas, ce délai « préthérapeutique » est une chance, encore faut-il l'expliciter de façon claire à la patiente ;

- l'autre question est plus perfide : dans quel cas actuellement un GS porteur de micro- ou macrométastase peut ne pas être synonyme de reprise de curage ? (résultats des essais connus et malgré des essais en cours comme SERC) Cela peut poser la délicate question d'un curage excessif sur une ponction ganglionnaire positive !

Ainsi, les situations extrêmes sont simples : les cellules isolées ou, à l'inverse, l'atteinte franche d'un étage de BERG ne posent pas de question philosophique. Il en va tout autrement de ces situations intermédiaires, pour lesquelles se retrouve autour d'une table chaque spécialiste, et chacun de proposer une adaptation : le chirurgien se positionnant sur une non-reprise, l'oncologue de ce fait revoyant une désescalade tentante mais dans le contexte excessif, le radiothérapeute arrivant après ses collègues n'ayant plus qu'à s'adapter à la situation, compensant une désescalade par 
une potentielle augmentation prudente des champs. Qui n'a pas été témoin de ces discussions de RCP pour ne pas croire à la complexité de ces équilibres successifs ?

Ainsi évoluent les savoirs par compagnonnage et expertise : l'exemple du second traitement conservateur, chez une femme traitée antérieurement par mastectomie partielle et radiothérapie, en est une belle illustration. Là encore, échanges préthérapeutiques entre chirurgiens et radiothérapeutes, qualité et précision des données permettent de renverser certains dogmes. La sagacité et l'évaluation permanente des traitements, leur suivi et la place de plus en plus prégnante du retour patient sur son ressenti et son rôle d'acteur dans la prise en charge sont les clés d'un quotidien passionnant, car sans cesse en mouvance, condamnant tout dogmatisme.

Bien des questions demeurent, au premier rang desquelles les récidives à long terme et leur prédictivité. Les propositions d'allongement de temps de traitements se heurtent non seulement à l'adhésion des patientes, pendant des années précieuses de leur vie, mais surtout à la difficulté de prédire avec justesse qui récidivera ou non. Depuis des décennies, la place de CTC est débattue à travers les retours d'expertises des équipes. L'heure est-elle venue du diagnostic d'une potentielle récidive ou d'un surrisque réel pour les femmes dites à risque familial ?

Depuis au moins aussi longtemps, la rechute sous traitement antihormonal désespère patientes et oncologues, et ce, malgré des progrès indéniables faits dans la compréhension des mécanismes, aussi complexes soient-ils ; au rang des nouvelles espérances, les mutations du gène ESRI occupent une place prometteuse.

Il n'en reste pas moins que le plus grand pari des années à venir reste la maitrise des traitements des tumeurs triplenégatives que redoutent aussi bien les patientes, ayant découvert avec effroi sur internet cette appellation réductrice, que les oncologues qui cherchent la voie. Sans se focaliser sur les mutations BRCA, quels sont les espoirs à venir en 2019 ? C'est peut-être là qu'enfin la cancérologie mammaire va pouvoir profiter des progrès de l'immunothérapie si prometteuse dans d'autres localisations.

Ces diverses réflexions devraient trouver des axes de réponses dans ce numéro forcément très sélectif et ne permettant pas de faire le point de toutes les actualités sénologiques.

Bien d'autres questions sont sujettes à réflexion... « de la suspicion » évoquant les stratégies de dépistage et de prévention à « l'après » qui est un véritable enjeu démographique et collaboratif.

Car la maladie n'est qu'un temps et illustre le passage de témoins entre les patients d'un temps et les biens portants, les médecins du quotidien et les spécialistes que les malades vont croiser de façon de plus en plus sporadique dans le cadre des thérapeutiques ambulatoires qui bouleversent bien des équilibres dans la relation ville-hôpital, dans un chemin de vie qu'il faut viser à optimiser, tous ensemble.

Bonne lecture! 